\title{
Neuromodulation influences synchronization and intrinsic
}

\section{read-out [version 1; peer review: 2 approved with}

\section{reservations]}

\author{
Gabriele Scheler (iD
}

Carl Correns Foundation for Mathematical Biology, Mountain View, CA, 94040, USA

V1 First published: 14 Aug 2018, 7:1277

https://doi.org/10.12688/f1000research.15804.1

Latest published: 02 Dec 2018, 7:1277

https://doi.org/10.12688/f1000research.15804.2

\section{Abstract}

Background: The roles of neuromodulation in a neural network, such as in a cortical microcolumn, are still incompletely understood.

Methods: (a) Neuromodulation influences neural processing by presynaptic and postsynaptic regulation of synaptic efficacy. Synaptic efficacy modulation can be an effective way to rapidly alter network density and topology. We show that altering network topology and density, will affect spike synchronization. Fast synaptic efficacy modulation may therefore influence the amount of correlated spiking in a network. (b) Neuromodulation also affects ion channel regulation for intrinsic excitability, which alters the neuron's activation function.

Results: We show that synchronization in a network influences the read-out of these intrinsic properties. Highly synchronous input drives neurons, such that differences in intrinsic properties disappear, while asynchronous input lets intrinsic properties determine output behavior. Thus, altering network topology can alter the balance between intrinsically vs. synaptically driven network activity.

Conclusion: We conclude that neuromodulation may allow a network to shift between a more synchronized transmission mode and a more asynchronous intrinsic read-out mode. This has significant implications for our understanding of the flexibility of cortical computations.

\section{Keywords}

neuromodulation, synchronization, network topology, synaptic efficacy, intrinsic excitability, asynchronous, activation function

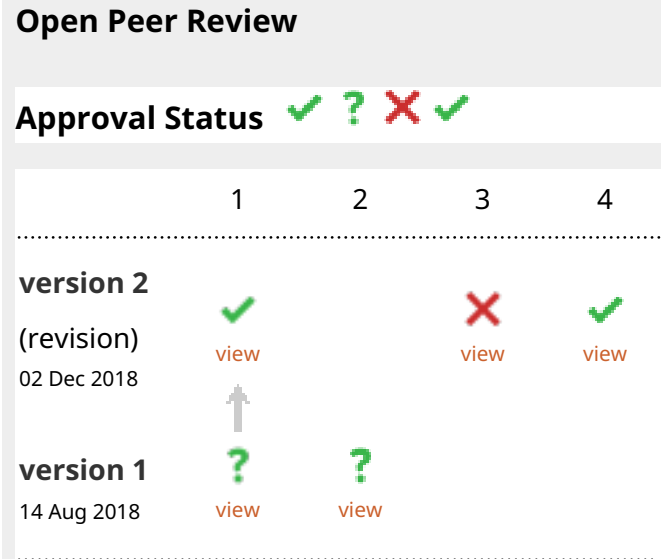

1. Joel Zylberberg, University of Colorado School of Medicine, Aurora, USA

2. Guillaume Drion (D), University of Liege, Liège, Belgium

3. Krešimir Josić, University of Houston, Houston, USA

4. Jonathan P. Miller, Case Western Reserve University, Cleveland, USA

Any reports and responses or comments on the article can be found at the end of the article. 
Corresponding author: Gabriele Scheler (gscheler@gmail.com)

Author roles: Scheler G: Conceptualization, Data Curation, Formal Analysis, Investigation, Methodology, Software, Validation, Visualization, Writing - Original Draft Preparation, Writing - Review \& Editing

Competing interests: No competing interests were disclosed.

Grant information: The author(s) declared that no grants were involved in supporting this work.

Copyright: ( $) 2018$ Scheler G. This is an open access article distributed under the terms of the Creative Commons Attribution License, which permits unrestricted use, distribution, and reproduction in any medium, provided the original work is properly cited.

How to cite this article: Scheler G. Neuromodulation influences synchronization and intrinsic read-out [version 1; peer review: 2 approved with reservations] F1000Research 2018, 7:1277 https://doi.org/10.12688/f1000research.15804.1

First published: 14 Aug 2018, 7:1277 https://doi.org/10.12688/f1000research.15804.1 


\section{Introduction}

In this paper we present a realistic network model, akin to a cortical microcolumn ${ }^{1-4}$, and investigate its properties under the assumption of fast synaptic and intrinsic modulation as evidenced by neuromodulation ${ }^{5}$. We hypothesize that rapid synaptic efficacy changes allow a network to operate with different topologies, and that network topology is a decisive factor towards creating and sustaining synchronized inputs vs. producing asynchronous input.

We have previously shown for a conductance-based neural model of striatal medium spiny neurons that neuronal heterogeneity expressed by the contribution of individual ion channels (such as delayed rectifier potassium channels or GIRK channels) may still result in uniform responses, if the neurons are driven with highly correlated synaptic input. If the same neurons are driven by more asynchronous, distributed synaptic input, the heterogeneity is manifest in the response patterns, i.e. the spike rates and the timing of the spikes (see 6). These results were achieved using conductance-based point neurons ${ }^{6}$. Here we use two-dimensional neural models ${ }^{7}$ to further investigate the effect and determine its significance in the context of a cortical neural network.

Due to Hebbian learning 8,9 , under normal conditions synaptic weights follow a lognormal distribution, which results in graphs with a heavy tail degree distribution. Degree modification by rapid synaptic efficacy changes would not only allow for alterations to the density, but also the topology of the connecting graph. In this paper we examine the hypothesis that such changes in network topology actually occur, driven by neuromodulatory effects on presynaptic release or postsynaptic response ${ }^{5,10-12}$. We analyze this situation with two example graphs, and we also perform further analysis to show that there is a continuum of graphs which can be reached by rapid synaptic changes.

\section{Methods}

\section{Conductance-based neuron model and synaptic input}

The conductance-based neural model of a striatal medium spiny neuron is described in detail in 6 . The membrane voltage $V_{m}$ is modeled using the equation

$$
\dot{V}_{m}=-\frac{1}{C}\left[\mu_{1}\left(I_{1}\right)+\mu_{2}\left(I_{2}\right)+\ldots+\mu_{n}\left(I_{n}\right)-I_{s y n}\right],
$$

where the $I_{i}$ are the currents, induced by the individual ion channels. Variability of the neuron is modeled by modifications to $\mu_{i}$. This model includes ion channels for $\mathrm{Na}$ (INa), $\mathrm{K}$ (IK), slow A-type $\mathrm{K}$ channels (IAs), fast A-type $\mathrm{K}$ channels (IAf), inward rectifying $\mathrm{K}$ channels (IKir), L-type calcium channels (ICaL), and the leak current $\left(\mathrm{I}_{\text {leak }}\right)$. The definition of all parameters and the dynamics of the ion channels can be found in 6.

For the experiments in this paper, we only focus on variability induced by changes in the strength of the slow A-type $\mathrm{K}$ channels. The total current contribution for this channel is $\mu_{\text {IAs }}$ where $\mu$ was selected between 1.0 and 1.5 .
In order to illustrate the variability in neuron behavior, we excited the neuron model by input signals, resembling two kinds of synaptic input: uncorrelated and correlated. These signals were generated by superposition of excitatory and inhibitory spikes from individual Poisson-distributed spike trains (50 excitatory and 10 inhibitory), and biased Gaussian background noise.

The amount of pairwise correlation in these spike trains governs the type of input signal. A high correlation factor was used in order to generate sequences which have short periods (10-15ms) of high activity.

\section{Heterogeneity in a two-dimensional model}

In order to do large-scale simulation we needed to employ a simple, computationally tractable neuron model. We used a two-dimensional model of a neural oscillator (cf. 7), and employed an instantiation of the model with parameters fitted to the general properties of cortical pyramidal neurons ${ }^{13}$ as a generic model $(\mathrm{g})$. The model consists of an equation for the membrane model $v$ (Equation 2), fitted to experimental values for cortical pyramidal neurons, and an equation for a gating parameter $u$ (Equation 3).

$$
\begin{gathered}
\dot{v}=0.04 v^{2}+5 v+140-u-I_{s y n} \\
\dot{u}=a(b v-u) \\
b=0.2 \\
a=0.02
\end{gathered}
$$

When the neuron fires a spike (defined as $v(t)=30 \mathrm{mV}$ ), $v$ is set back to a low membrane potential $v:=c ; c=-65.8 \mathrm{mV}$ and the gating variable $u$ is increased by a fixed amount $d(u:=u+d ; d=8)$ (cf. 13). This formulation allows for a very simple neuron model, which avoids the explicit modeling of the downslope of the action potential, and rather resets the voltage. Time-dependence is modeled by the gating variable $u$.

Neuronal heterogeneity is achieved by systematic variation of inactivation parameters. By varying $d$, we can vary the inactivation dynamics of the model after a spike, by varying $a$ we vary the inactivation dynamics throughout the computation. In this way, we can attempt to model neuronal variability in activation/ inactivation dynamics, which is sufficient to model frequencyselectivity as a stored intrinsic property. The parameters used in this paper for different neuron types are listed in Table 1.

\section{Graph properties}

We created graphs of $N(=210)$ excitatory neurons, and $K(\sim 1900)$ excitatory connections. For the excitatory neurons, we use randomly connected graphs $(\mathrm{N}, \mathrm{K})$ with different dispersion $\sigma^{*}$. This corresponds to normal (Gaussian) to lognormal graphs with different widths and length of the heavy tail. We model inhibition by Poisson-distributed inhibitory synaptic input directly onto excitatory neurons.

We use specific instantiations of these graphs (RG, LG1) for the simulations. Table 2 shows global graph characteristics for the 
Gaussian graph (RG), the lognormal graphs (LG1), and intermediate graphs LG2, LG3, and LG4.

\section{Definitions}

We define synchronization $s$ in a network by pairwise correlations: for each neuron $n_{i}$, we count, for each other neuron $n_{j}$, the number of spikes which occur within a window $W(W=10 \mathrm{~ms})$ of $n_{i}$ 's spike events, divided by the total number of spikes for $n_{i}$. More precisely, for each neuron, we bin all firing events into $5 \mathrm{~ms}$ bins. We then count the number of other neurons, which fire in a $10 \mathrm{~ms}$ window around the (start) of the bin. The synchronization $s$ is then the average over all neuron pairs in the network:

$$
s=\frac{1}{N(N-1)} \sum_{i} \frac{1}{S_{j}} \sum_{i} B_{i j},
$$

where $B_{i j}$ is the number of spikes that neurons $i$ and $j$ have in common within a moving window of $W=10 \mathrm{~ms}$ during the entire measuring time. $S_{j}$ is the number of spikes of neuron $j$ during the entire measuring time.

The rewiring algorithm used to change the properties of a graph $\mathrm{G}$ is a greedy algorithm, which iteratively selects the node $s$ with the highest degree. One of its edges is then rewired to random nodes with lower degrees, decreasing $\sigma^{*}$. The algorithm terminates, when the value of $\sigma^{*}$ falls below a given threshold.

\section{Simulation tools}

All simulations were performed with the software tool CNeuroSim, which is implemented in Matlab (R2016b) and C, and available at 14 .

\section{Results}

Conditional expression of intrinsic excitability in conductance-based models

We show how we can model gain as a stored intrinsic property, defined as a spike rate in response to constant or fluctuating input of fixed strength $(\mathrm{A} / \mathrm{Hz})$. We use a full ion channel based model (the MSN $\operatorname{model}^{6}$ ), with variation in the slow A-type potassium channel (IAs).
In Figure 1A, we show the response of MSN model neurons with a scaling of $\mu_{\mathrm{IAs}}=1.0,1.3,1.5$ to a noisy signal, derived from uncorrelated Poisson-distributed synaptic input. The top panel shows the development of the membrane potential, $V_{m}$, over time for all neurons. The middle panel shows the spike-train for each neuron with the mean ISI and its standard deviation; the total number of spikes is shown on the right. The total number of spikes includes bursts, which were excluded from ISI calculation. The bottom panel shows the synaptic input. The dots correspond to the spiking events for a single neuron $3\left(\mu_{\mathrm{IAs}}=1.5\right)$. The resulting mean ISIs are 25,37 , and $45 \mathrm{~ms}$. With a standard deviation of 6,11 , and 8 , they are clearly distinguishable. This is also shown by the Gaussian distribution for the mean ISIs for each neuron type (Figure 1B).

This model shows frequency-specificity as read-out of the relative contribution of the slow A-type potassium channel, indicated by the scaling factor $\mu_{\mathrm{IAs}}$. The relative contribution of an ion channel corresponds to its density or distribution on the somato-dendritic membrane, or in some cases its specific localization at dendritic branch points. Experimental evidence has shown that this is a plastic feature for neurons.

We then employ highly correlated synaptic input, defined as in 6 (see Methods). We stimulate the same neurons with the correlated input and observe the spike pattern (Figure 2). We can show that the frequency-specificity of the neuron disap-

Table 1. Parameters for different neuron types.

\begin{tabular}{|l|l|l|l|l|}
\hline name & $\boldsymbol{a}$ & $\boldsymbol{b}$ & $\boldsymbol{c}$ & $\boldsymbol{d}$ \\
\hline generic & 0.02 & 0.2 & -65 & 8 \\
\hline type1 & 0.025 & 0.2 & -65 & 6 \\
\hline type2 & 0.02 & 0.2 & -65 & 9 \\
\hline type3 & 0.015 & 0.2 & -65 & 12 \\
\hline type4 & 0.015 & 0.15 & -65 & 14 \\
\hline type5 & 0.022 & 0.3 & -65 & 14 \\
\hline type6 & 0.022 & 0.3 & -65 & 9.5 \\
\hline
\end{tabular}

Table 2. Graph Properties for Excitatory Connections.

\begin{tabular}{|l|l|l|l|l|l|}
\hline Property & RG & LG1 & LG2 & LG3 & LG4 \\
\hline $\mathrm{N}$ & 210 & 210 & 210 & 210 & 210 \\
\hline $\mathrm{K}$ & 1880 & 1924 & 1895 & 1920 & 2050 \\
\hline $\mathrm{d}$ & 0.042 & 0.043 & 0.043 & 0.043 & 0.047 \\
\hline indegree & $8.95[2 \ldots 17]$ & $9.5[0 \ldots 26]$ & $9[0 \ldots 24]$ & $9.14[0 \ldots 30]$ & $9.75[0 \ldots 25]$ \\
\hline outdegree & $8.95[4 \ldots 18]$ & $9.5[0 \ldots 56]$ & $9[0 \ldots 58]$ & $9.14[0 \ldots 53]$ & $9.75[0 \ldots 54]$ \\
\hline cluster index & 0.04 & 0.068 & 0.064 & 0.062 & 0.08 \\
\hline mean plength & 2.66 & 2.41 & 3.7 & 3.72 & 2.32 \\
\hline$\sigma^{*}$ & 1.44 & 2.89 & 2.5 & 2.71 & 2.71 \\
\hline synchron s & 0.11 & 0.32 & 0.26 & 0.31 & 0.34 \\
\hline
\end{tabular}




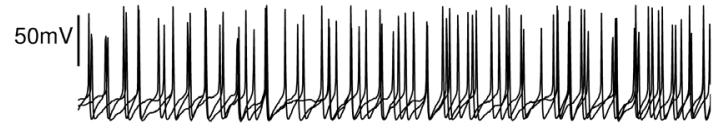

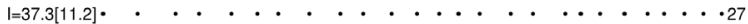

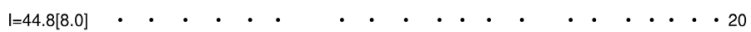

A

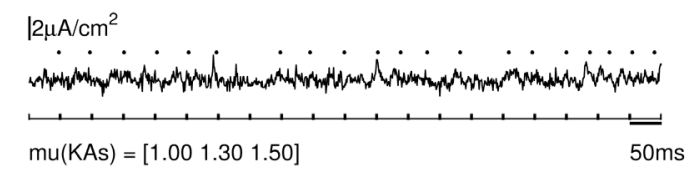

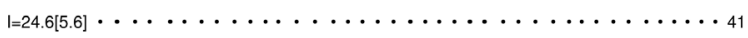

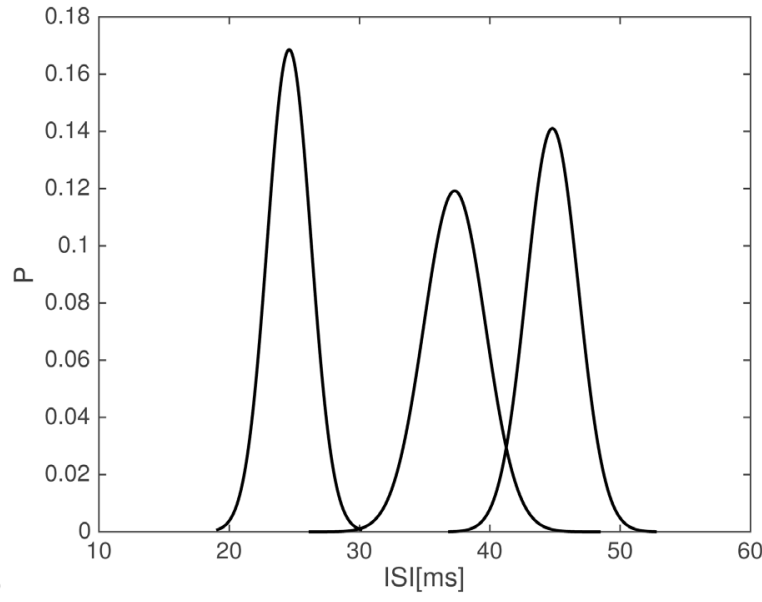

B

Probability

Figure 1. A. Frequency response of 3 conductance-based MSN model ne
distributions of ISIs. We see a clear separation of frequency responses.

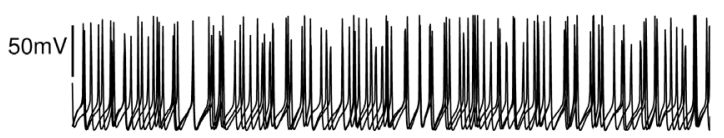

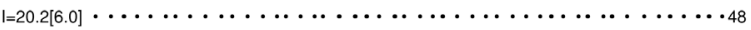

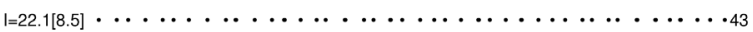

$\mathrm{l}=25.6[6.3] \ldots \ldots \ldots \ldots \ldots \ldots \ldots \ldots \ldots \ldots \ldots \ldots \ldots \ldots \ldots \ldots \ldots \ldots$

A
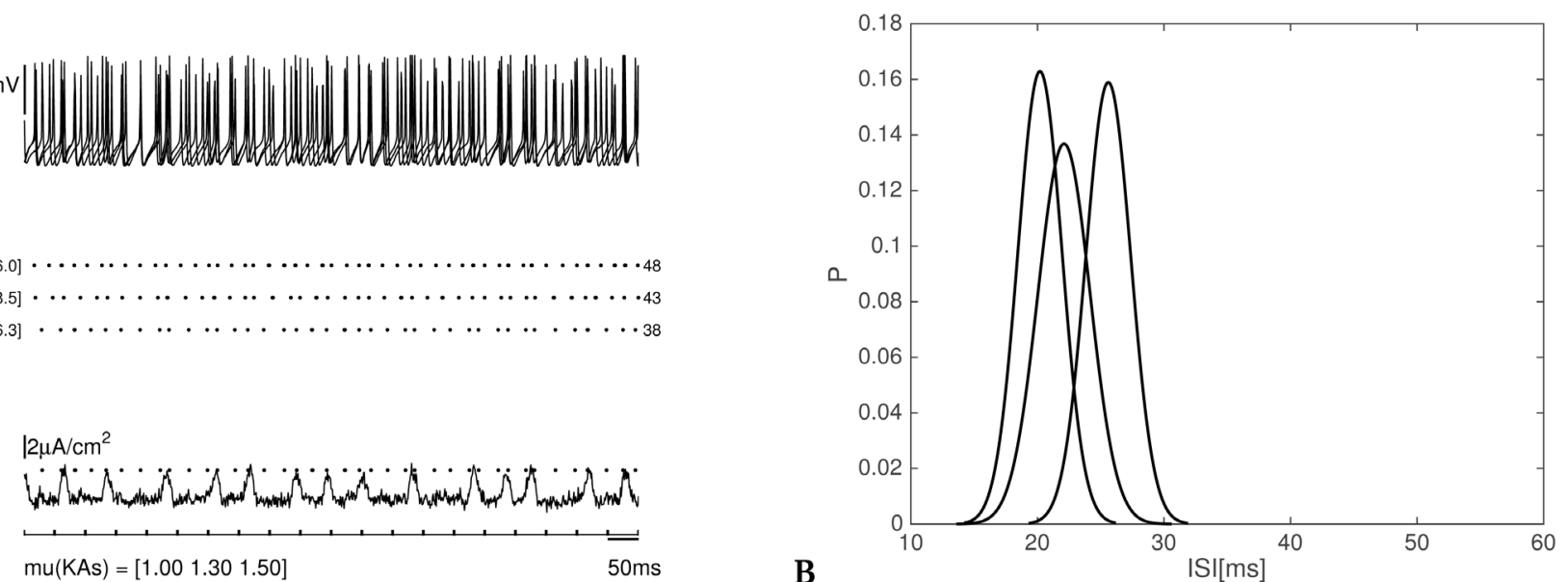

B

Probability distribution of ISIs. We Figure 2. A. Frequency response of the
see overlapping of frequency responses.

pears. Instead we see a time-locked spike pattern which is expressed by a similar spike frequency (Figure 2A) and an overlap of the mean ISIs (Figure 2B). What this experiment showed is that a stored intrinsic property, the gain, is available to the processing network in a conditional manner. The property is continually expressed, the differences in ion channel density persist. Depending on the mode of stimulation, however, this property is manifested as intrinsic gain, or it is obscured when a neuron is driven by strongly correlated input.

Results for simplified model neurons

To continue with exploring this property of model neurons in a networked context, we switched to a simplified model neuron $^{7}$ and created a set of variations for this model (see Meth$o d s)$. We show the response of two-dimensional model neurons to asynchronous input in Figure 3, and to regular, synchronous input in Figure 4. In the first case, we have clearly separated frequencies, and in the second case, the ISIs are nearly identical with a narrow distribution. When we stimulate the neurons with irregular, but synchronous input, the ISIs become identical, but with a wider distribution to reflect the different duration of pauses between the synchronous stimulation (Figure 5).

\section{Multiplexing synchronous and asynchronous input}

We may also consider the question of whether a neuron can simultaneously respond to an input and read out its stored spike frequency. If there are single synchronous events, which interrupt ongoing spiking, can we recover the intrinsic properties for each neuron? In Figure 6 it is shown that this is possible. Figure $6 \mathrm{~A}$ shows the input and the synchronous responses, and in Figure $6 \mathrm{~B}$ we still see a clear separation of frequencies. 


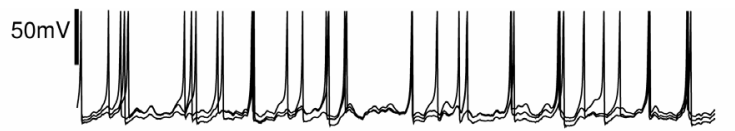

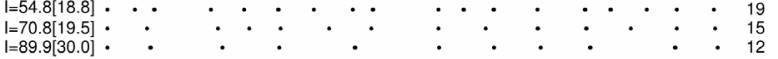

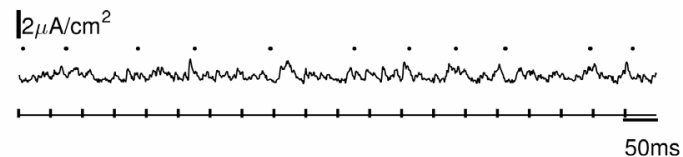

B

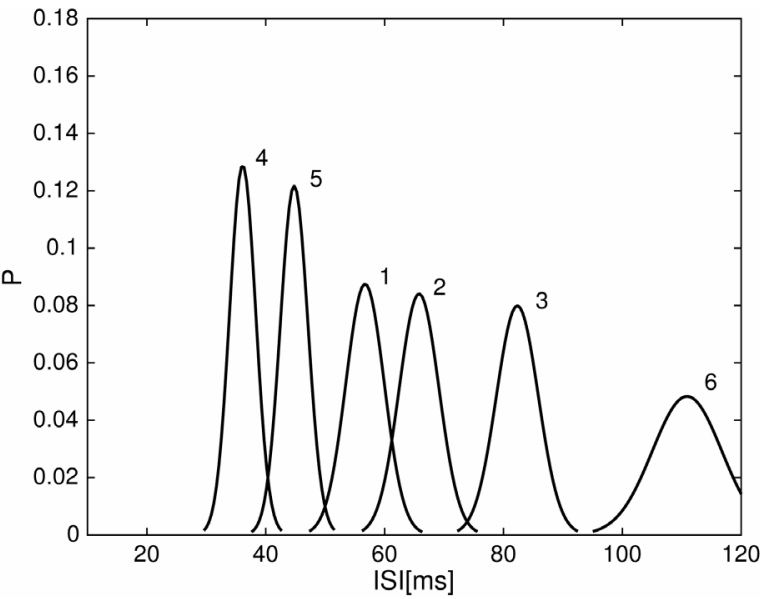

Figure 3. A. Spike response of the two-dimensional dynamic model neurons $(1,2,3)$ to asynchronous input. B. Gaussian distributions of ISIs for six model neurons $(1,2,3,4,5,6)$ to asynchronous input as in $\mathbf{A}$. We see a clear separation of frequency responses for model neurons.

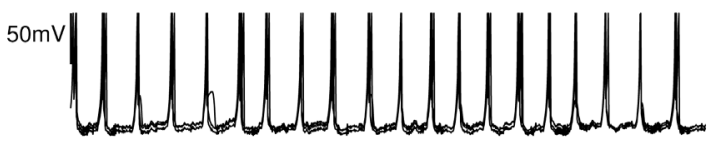

I=46.5[4.7]- -

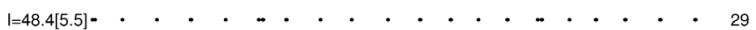

$\mathrm{I}=51.7[9.4]-\cdot . \cdot$. . . . . . . . . . . 23

A

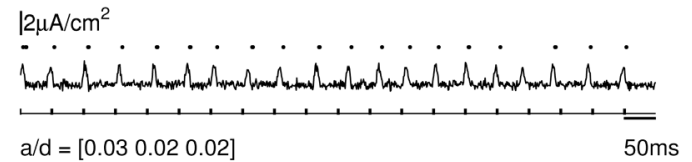

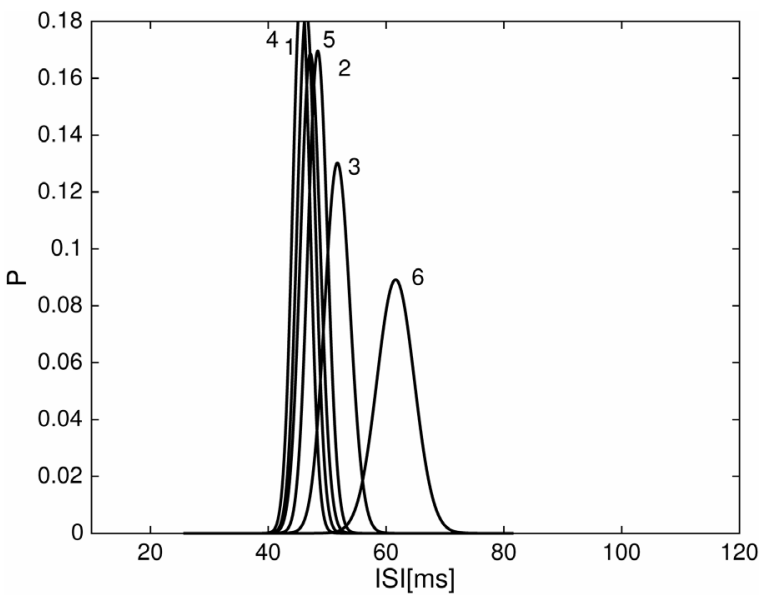

Figure 4. A. Spike response of the two-dimensional dynamic model neurons $(1,2,3)$ to regularly timed, correlated input. B. Distributions of ISIs for six model neurons $(1,2,3,4,5,6)$ to the same input as in $\mathbf{A}$. We see strong overlapping of frequency responses, at about $50 \mathrm{~ms}$ ISI, in accordance with the input. We notice that neuron 6 fires at lower frequencies than the input, it probably has a longer reset period, as seen in Figure 3B.
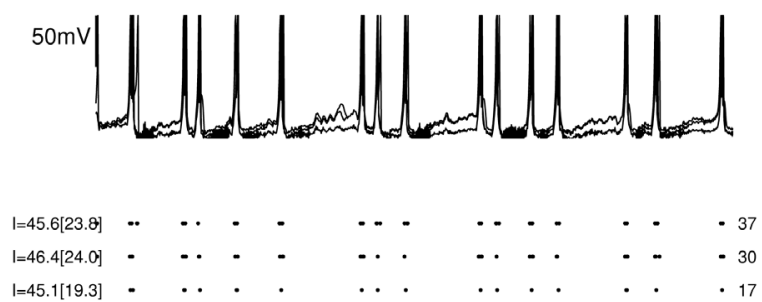

A

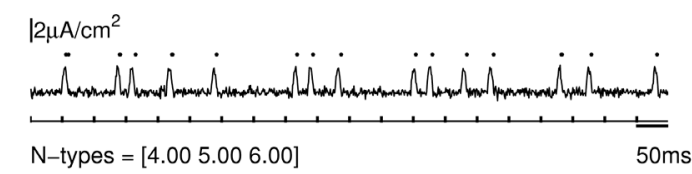

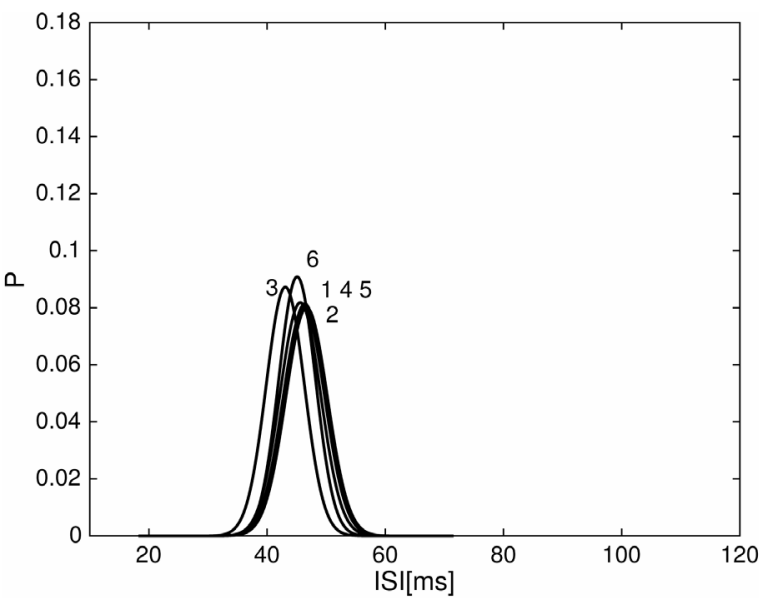

B

Figure 5. A. Spike response of the two-dimensional dynamic model neurons $(1,2,3)$ to irregularly timed, correlated input. B. Gaussian distributions of ISIs for six model neurons $(1,2,3,4,5,6)$. We see strong overlap of frequency responses. 

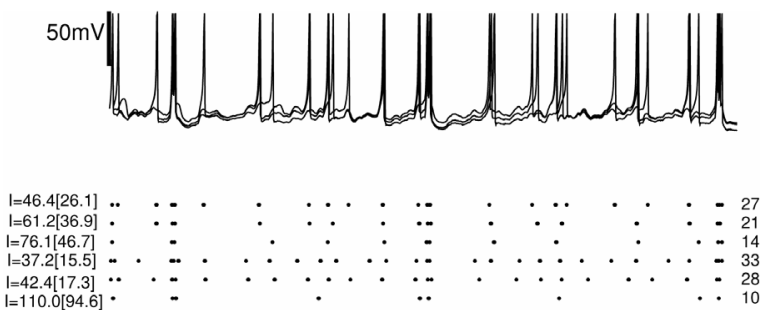

A

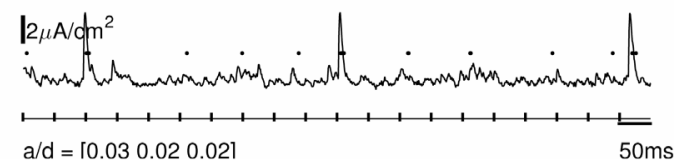

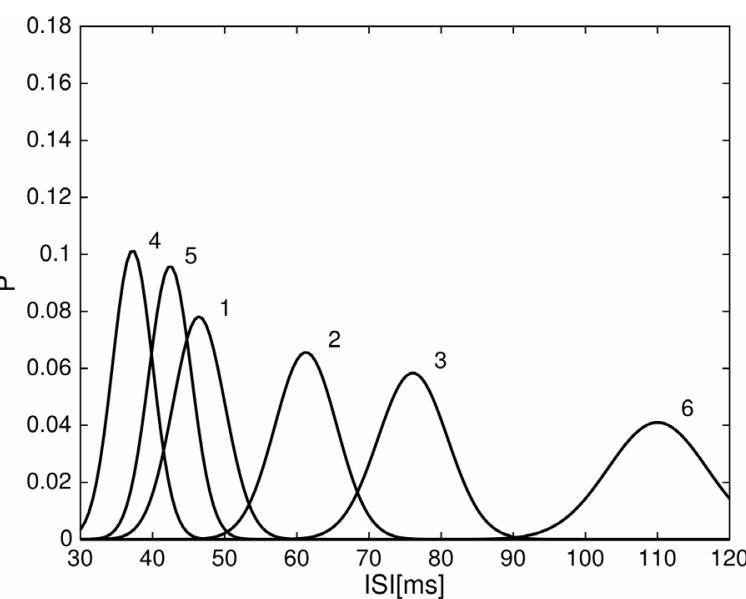

Figure 6. A. Multiplexed input and response of different model neurons (1-6). Three synchronous events are clearly represented in the spike pattern of all neurons. B. Mean ISIs for each neuron type. The separation of frequencies is kept. Compared to Figure 3B the standard deviation is somewhat higher because of the additional spikes caused by strong synchronous input.

We conclude that we can multiplex asynchronous and synchronous input. It is also apparent that there needs to be a lower limit on the intervals between synchronous events that can be processed without disrupting intrinsic properties. This interval needs to be defined as functionally dependent on the intrinsic frequencies. In this case, it is $3 / \mathrm{s}$ for the synchronous events, with $10 \mathrm{~Hz}$ for the slowest neuron.

\section{Synchronization depends on network topology}

The simplified model neurons allow the creation of large networks of heterogeneous neurons and exploration of different topologies. We hypothesized that a lognormal graph, because of its hierarchical topology and the existence of hub neurons would lead to synchronization of action potentials - even with heterogeneous neurons - while a Gaussian topology would support asynchronous spiking behavior ${ }^{15,16}$. We define synchronization $s$ in a network by pairwise correlation (Methods). The spike frequency for each neuron type is assessed by the mean and standard deviation for ISIs, as before.

We first use a Gaussian connected graph (RG) with $N=210$ and $K=1800$ and use 7 different neuronal types (1-6, plus the generic neuron g) with 30 Neurons each (Methods). Figure 7A shows an excerpt of the graph structure. We can see that the graph is connected such that all neurons have a comparable number of connections. This is also apparent in Figure 8, where we can see a (narrow) normal distribution for connectivity for the Gaussian graph RG. Table 2 contains the graph characteristics for both graphs.

$N=210$ is about the size of a minicolumn or ensemble unit within a larger network with presumably dense interconnections ${ }^{17}$. The maximal density $d=K /(N \times N-1)$ in a cortical microcircuit is estimated at 0.1 for $10^{4}$ neurons, and $10^{7}$ synaptic connections, ${ }^{18}$. With $~ 50 \%$ of synapses internal to the network, $d=0.04-0.07$ is a realistic value for internal connectivity ${ }^{17}$. There is also a small background inhibition to all neurons present, implemented by $10 \%$ inhibitory neurons with Poisson-distributed firing and complete connectivity to excitatory neurons.

We now stimulate the graph by an initial stimulation to 10 excitatory neurons (for about 1s). In Figure 9A, we see highly asynchronous neuronal activity after $1 \mathrm{~s}$ of stimulation. The pairwise correlation values $s$ is low $(s=0.11)$. Figure 9B shows that each neuronal type retains its own frequency, i.e., has its own typical ISI, separated from other neuronal types. We also notice that some neurons fire with low frequencies $(5 \mathrm{~Hz})$ and others with higher frequencies $(20 \mathrm{~Hz})$. Very low firing neurons $(2 \mathrm{~Hz})$ which are typical for cortex are not represented in this model.

Next we changed the topology of the network to a lognormal graph (LG1), with $N=210$ and $K=1924$ and used the same neurons as before.

Figure 7B shows an excerpt of a lognormal graph structure. The connectivity structure seems much denser, because of 'hub' neurons in the center of the graph. In Figure 8, we can see a much wider distribution of degrees for the lognormal graph (blue), with a number of nodes with high connectivity. Presumably, those nodes are capable of synchronizing the network, because they can reach many neurons simultaneously. What is the effect on the presence of neural heterogeneity?

Figure 10 shows that a high amount of synchronization can be achieved in spite of heterogeneity of intrinsic frequency of model neurons. The rasterplot (Figure 10A) shows the activity in LG1 with the same neurons and the same stimulation as before. The overall correlation, defined by pairwise correlation of neurons, is much higher $(\mathrm{s}=0.32)$. The distribution of ISIs in this case is strongly overlapping (Figure 10B), similar to Figure 4B, where neurons were explicitly driven by highly synchronous input. This means that synchronization is dependent on the network topology, and a lognormal graph exhibits a higher 
A

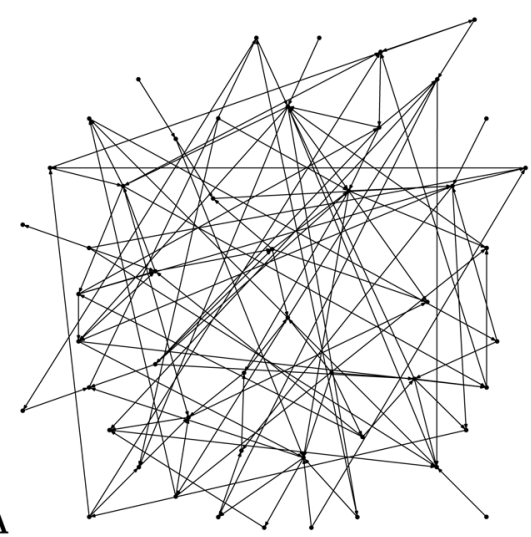

B

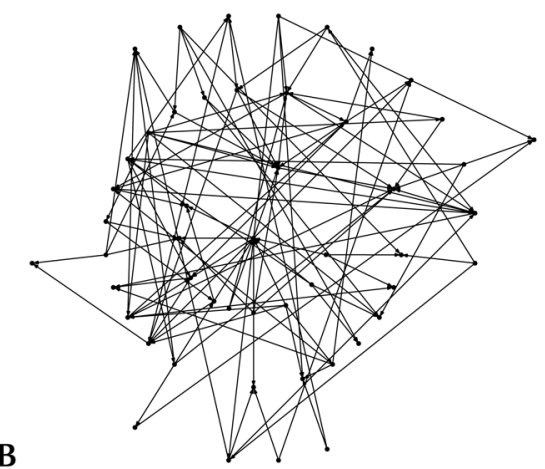

Figure 7. A. Part of a Gaussian Graph (RG), here for 50 neurons B. Part of a Lognormal Graph (LG1), for 50 neurons. The more regular, lattice-like structure of the Gaussian graph and the higher clustering and the appearance of highly connected 'hub' neurons in the lognormal graph is apparent.

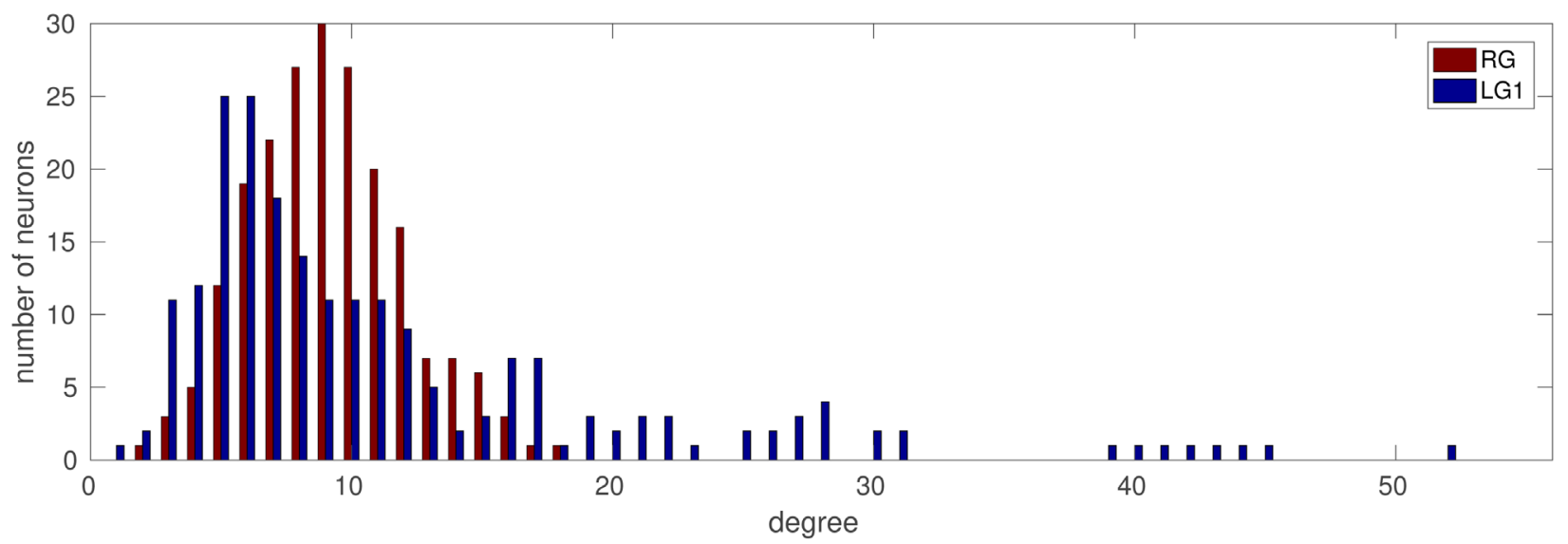

Figure 8. Degree histogram for the Gaussian Graph RG (red) and the lognormal Graph LG1 (blue). The LG has more neurons with few connections. It also has a heavy tail of neurons with 20 and more connections ('hubs'), which are lacking in the Gaussian graph.
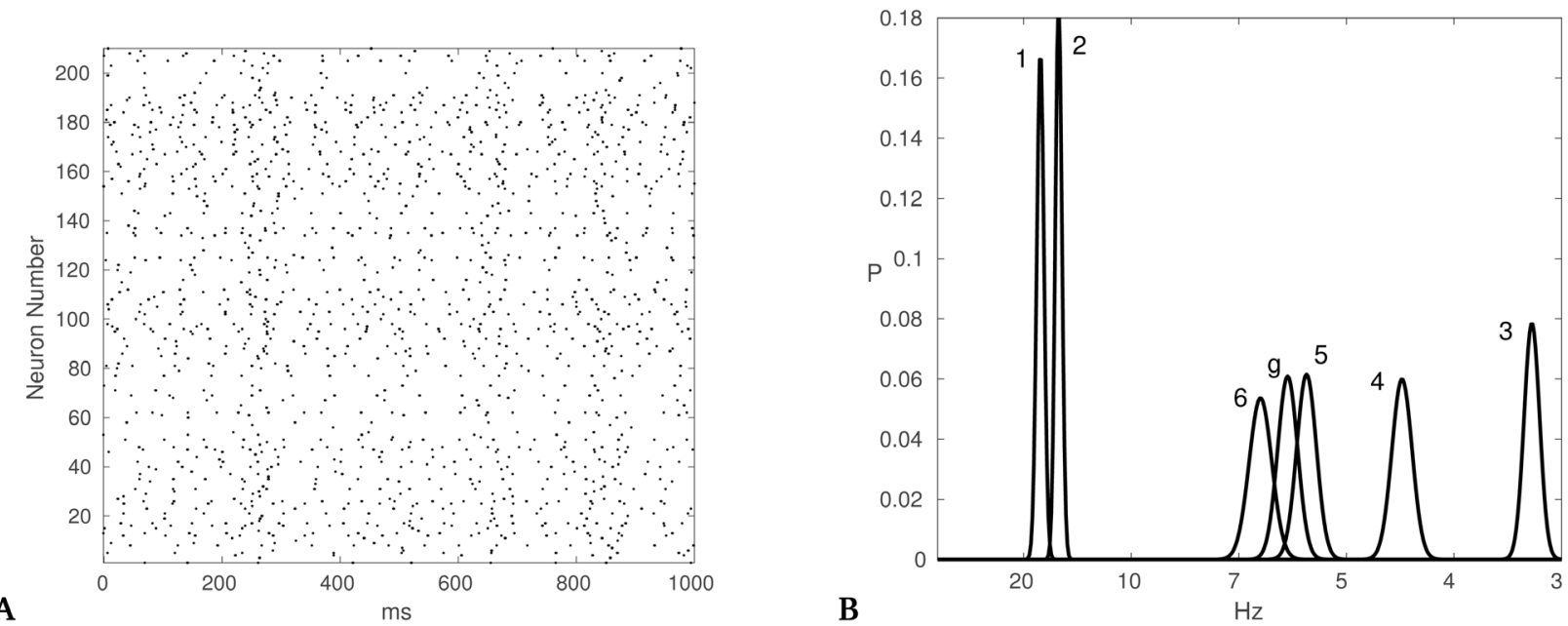

Figure 9. A. Asynchronous behavior in the Gaussian graph with variable neuron types. Groups of neuronal types are apparent in the rasterplot. Some structure is probably due to background inhibition. B. Average spikes/s with probability distributions for all neuronal types, with clear separation by frequency. Pairwise correlation is $s=0.11$. 

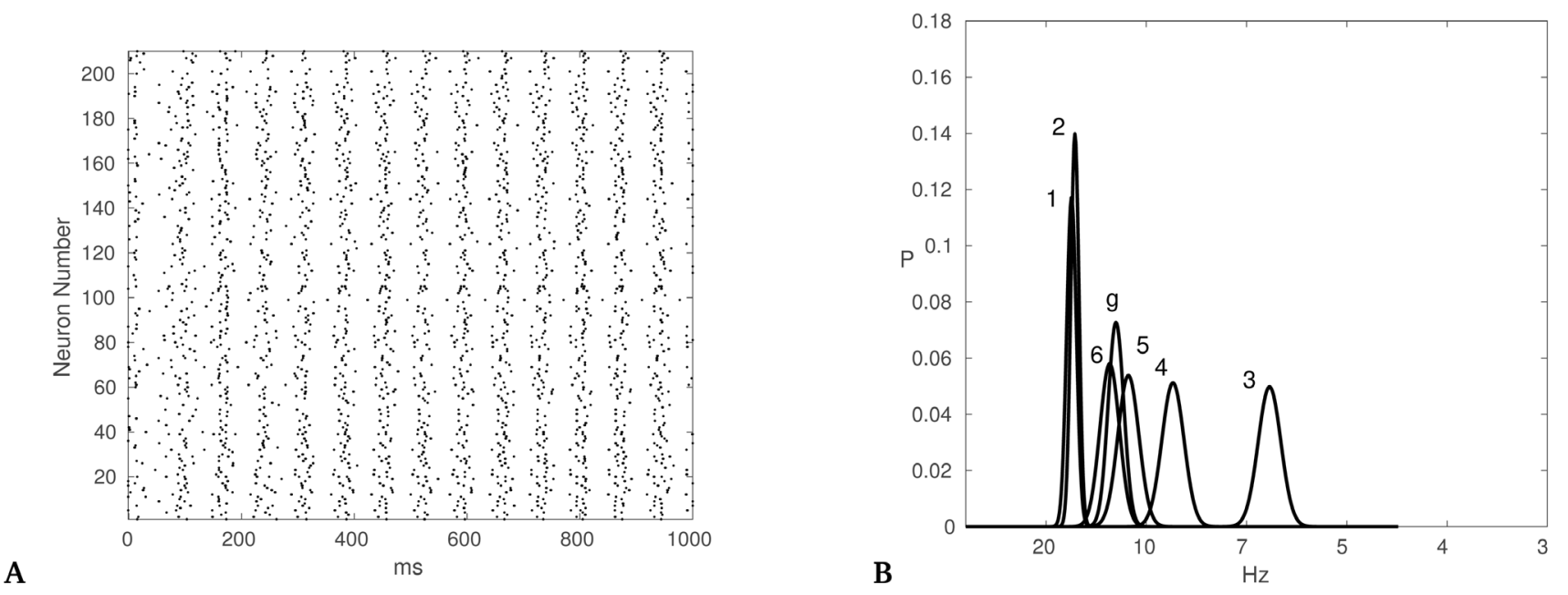

Figure 10. A. Synchronization in a heavy-tail graph (LG1) with variable neuron types. The rasterplot shows that different neuronal types respond uniformly. B. Frequency distributions. High overlap between neuronal types is apparent. Pairwise correlation in the graph is high with $s=0.32$.

tendency for pairwise synchronization. Also, that neuronal heterogeneity is apparent in an asynchronous network mode but is repressed in a synchronous firing mode.

\section{Dependence of synchronization on graph properties}

We could show that differences in intrinsic properties appear or become more prominent when there is less synchronicity in a network. In our model, the pairwise synchronicity $s$ is dominated by the network topology, more precisely by the width of the degree distribution (dispersion) ranging from Gaussian to lognormal with a heavy tail.

To confirm this observation we used a number of intermediate graphs and mapped the pairwise synchronization dependent on the dispersion $\sigma^{*}$ (Figure 11). The graphs RG and LG1 that we used have values of $\sigma^{*}=1.44$ and $\sigma^{*}=2.89$ (Methods). They have the same density, i.e., the same number of connections and neurons (0.05). Additionally, we analysed the dependence of synchronicity on the density of the graph between 0.01 and 0.1 (Figure 11).

There is higher synchronization in the lognormal region, especially with higher $\sigma^{*}>2.5$ but no synchronization for Gaussian graphs. For heavy-tail graphs, synchronization depends linearly on the density between $d=0.03-0.08(s=0.2-0.5)$.

How are the different graphs related? We hypothesized that fast synaptic switching ${ }^{19}$ by neuromodulation could change the network topology sufficiently to switch from a synchronous to an asynchronous regime. In Figure 12, we plot the number of edges that were changed to achieve different dispersions $\sigma^{*}$ of a graph. The algorithm used was a simple greedy algorithm (Methods), which is suboptimal, i.e. overestimates the number of edges required. It appears that $30-50 \%$ of edges changed would be sufficient.

\section{Discussion}

Network Topology, Synchronization and Intrinsic Read-out We employ a parameterizable two-dimensional neural oscillator model to encode different intrinsic excitability manifested by different frequency responses to constant input. What the experiments show is that a stored intrinsic property, the gain, is available to the processing network in a conditional manner: The gain is continually present, the differences in ion channel density persist. Depending on the mode of stimulation, however, this property is manifested as spike rate, or it is obscured when a neuron is driven by strongly correlated input. This is interesting because it shows a property of memory that synaptic plasticity lacks: the memory is not always 'read-out' in any processing step. It is conditional, it can be accessed or ignored depending on the state of the network. This seems to be an essential property of memory in any intelligent system.

Different statistical properties of synaptic input can be modeled by a variability in the correlation properties of input neurons. In a network model, this means that the overall correlation in the network determines what input a neuron receives. With a Gaussian degree distribution topology, correlation is low and neurons fire irregularly with their own preferred frequency. With a heavy-tailed, lognormal topology, correlation is higher, and neurons fire when they receive correlated input, irrespective of intrinsic properties. I.e. driving neurons by correlated vs. uncorrelated input leads to uniform spiking behavior vs. read-out of stored differences in ion channel conductances.

\section{Inhibition}

A restriction of the present model with respect to a biological simulation model is the simplified treatment of inhibition. However, experimental work shows that cortical parvalbuminexpressing $(\mathrm{PV}+)$, fast-spiking interneurons have no connection specificity to pyramidal neurons, rather they present as an 


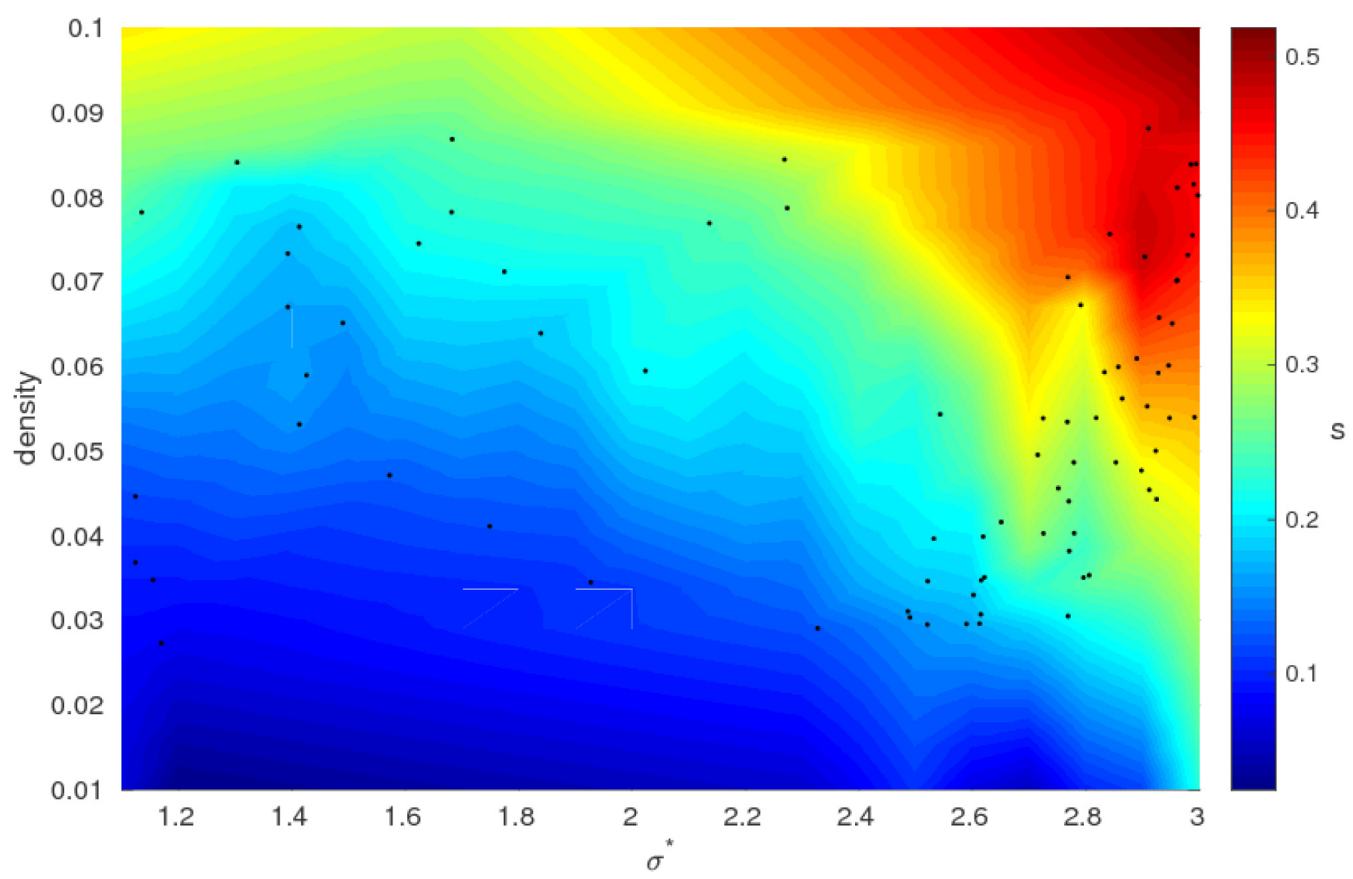

Figure 11. Synchronization $\mathbf{s}$ dependent of network topology: density and dispersion. The experimentally attested dispersion for weights in cortical tissue is $\sigma^{*}=2-3.5$, with a mean at 3 . We achieve higher synchronization in the lognormal region, also dependent on density, but no synchronization in the Gaussian (low dispersion region) $\left(\sigma^{*}<1.5\right)$, except close to maximal connectivity. Black dots signify actual measurements. There seem to be no abrupt transitions.

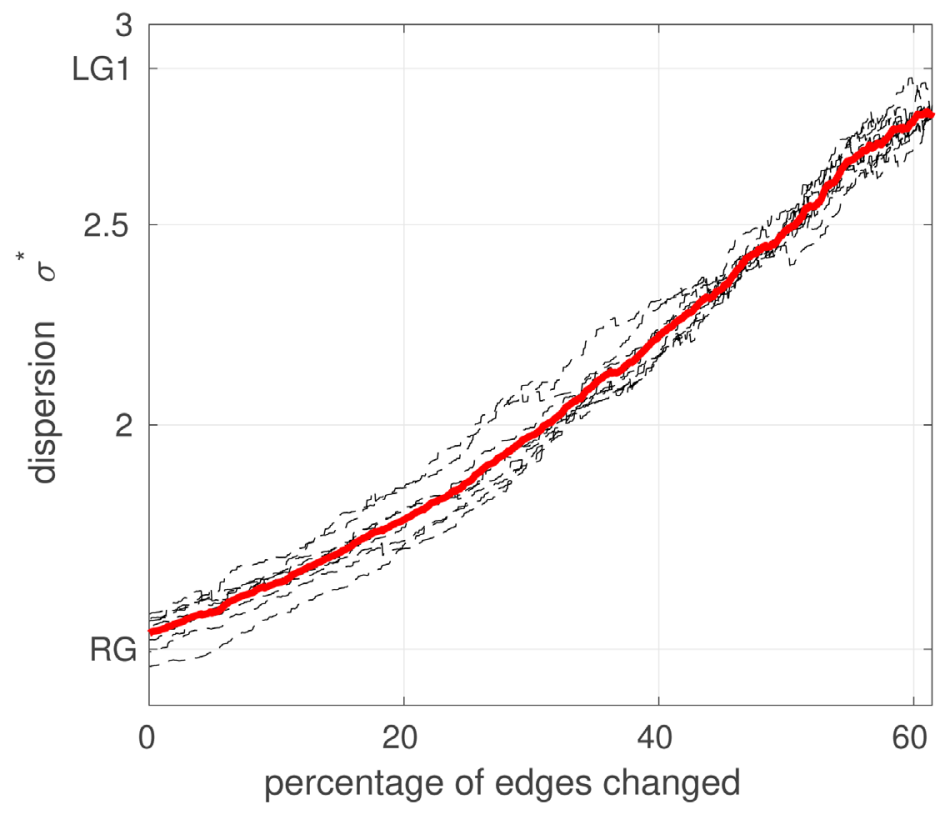

Figure 12. Transition between a lognormal graph and a Gaussian graph: For $K=1900, N=210, d=0.43$, mean over 10 trials, the percentage of edges changed to achieve a narrow degree distribution. The algorithm is not optimized (Methods) and overestimates the number of edges that have to be changed. 
'unspecific, densely homogeneous matrix covering all nearby pyramidal cells' (20, p. 13260), which corresponds to our model.

Conditions for neuronal read-out may include the activity of inhibitory neurons. Inhibition and excitation are tightly linked by feedback interaction. 21 suggested that the close coupling of inhibition and excitation in cortical tissue cancels out purely input-dependent, i.e. not network generated synchrony. 13 suggested that with highly correlated input, both inhibitory and excitatory, the neuron may receive less input which allows it to be driven only by strong synaptic input, with distributed input it may receive a barrage of excitatory and inhibitory inputs where the membrane voltage remains close to firing threshold and the neuron fires continuously. In our sense, it is reading out stored properties. Both inhibitory and excitatory synaptic input will conform to be asynchronous or synchronous, to drive neurons or it causes a neuron to emit spikes according to its own stored intrinsic properties.

However, neuromodulation has effects on inhibitory neurons as well ${ }^{22,23}$, which we have not modeled. Further simulations will show whether the I-E coupling is altered during enhanced neuromodulation, or whether the effects are synergistic to the present results.

\section{The role of neuromodulation}

Neuromodulation influences both intrinsic properties and synaptic connectivity $^{5}$, e.g. acetylcholine, (via nucleus basalis stimulation), noradrenaline (via LC stimulation) or dopamine (via VTA stimulation $)^{5,24}$. Experimental estimates on the distribution of synaptic neuromodulatory receptors are at approximately $30 \%-50 \%$ of connections ${ }^{19}$. That is sufficient to transform the topological properties of a graph, such as the dispersion of its degree distribution from heavy-tailed graph to a more Gaussian, less clustered graph without requiring tight optimization for the positions of neuromodulatory receptors (Figure 12).

Neuromodulation disables or enhances various ion channels, such as Sk-channels which guide reset times after a spike, or A-type potassium channels which influence latency to spike ${ }^{6}$. In this way, neuromodulation influences intrinsic properties. If neuromodulation reduces synchrony by acting at synaptic receptors, it uncovers intrinsic heterogeneity, and induces a mode of processing that allows read-out and storing of intrinsic properties. Depending on the neuromodulator used, and the amplitude and duration of the signal, different soma-dendritic ion channel profiles would emerge.

In the synchronous mode, intrinsic heterogeneities are reduced in the presence of tightly correlated input which drives neurons reliably. This invariance of neuronal intrinsic properties in synchronous mode allows synaptic transmission and information processing independent of neuronal heterogeneity.

The idea of introducing synchronous events by common input to an asynchronous background, and in this way use reliable synaptic transmission without affecting the state of the system (multiplexing) has also been documented in experimental results. For instance, (25, Figure 4A) shows a case of multiplexing in response to behavioral stimuli. In this case, intrinsic read-out can continue, and single events are transmitted reliably through driven activations.

Why should synchronization properties be switched by neuromodulation? Increased correlation in the network supports population-coded information to be propagated effectively ${ }^{26}$. Turning on neuromodulation would decorrelate an area and increase the capacity for information coding in an ensemble or a cortical microcolumn ${ }^{27}$. This area would become an information source to surrounding areas. When turned off, increased correlation would allow this area to transmit information and to disregard the stored neural memory.

\section{Relation to experimental evidence}

Basal forebrain stimulation, which results in increased acetylcholine release and muscarinic/nicotinic receptor activation, decreases correlation between cortical neurons (28-30 (Figure 3.C)). Likewise, 31 (Figure 3 and Figure 4) shows reduced noise (internal) correlations with cholinergic stimulation, while inactivation of the basal forebrain caused more synchronized activity. 32 shows reduction of correlation for task-relevant perception, where presumably task-relevance causes neuromodulatory activity. 33 provides evidence for the involvement of noradrenaline in desynchronization of cortical state and the enhancement of sensory coding.

There is considerable evidence ${ }^{34-37}$ showing that several neuromodulators, including at least noradrenaline and acetylcholine, modulate pairwise spike correlation, such that strongly synchronized states (anesthesia, slow wave sleep) have high correlation and low neuromodulation, while asynchronous states (normal waking), with higher neuromodulation, have lower pairwise correlation.

37 observed intrinsic fluctuations in synchronization of cortical networks during wakefulness which correlated with the amount of encoded perceptual information and perceptual performance. Their results showed a mean decrease in correlations from synchronized to desynchronized state corresponding to perceptual performance by approximately $20 \%$, similar to values observed during attention ${ }^{38}$, and after adaptation ${ }^{39}$. We have shown (Figure 11) that correlation changes are continuous with network topology and a $20 \%$ correlation change is well within the range of the current simulations. Importantly, the results in 37 point to fluctuations in synchronization that reflect local changes in network activity rather than just global cortical state dynamics which have traditionally been associated with central neuromodulatory release.

The role of presynaptic neuromodulation in suppressing cortical connections ${ }^{11,12}$ and changing attractor states ${ }^{40}$, as well as allowing rapid synaptic weight changes ${ }^{19}$ has previously been assessed. Theoretical work has also emphasized the connection between correlations and information content ${ }^{27,41-43}$.

Here we bring these observations together to suggest that neuromodulation of synapses may alter network topology and in this way bring about an increased decorrelation of spiking, and a more asynchronous state, with a higher informational capacity. 
It may provide a general explanation (a) on how fluctuations in synchrony can be engineered rapidly and in small cortical areas and (b) why intrinsic memory may be conditional, accessible only at certain times and in a localized fashion.

\section{Conclusion}

We created a number of different parameterized neuron models to capture neuronal heterogeneity. This affects the properties of the neuron such that it has less or more intrinsic excitability, leading to different firing rates when stimulated in an asynchronous way. Under synchronous stimulation the differences are greatly reduced.

We also suggested that synaptic neuromodulation can be an effective way of rapidly altering network topology. We investigated changes in network topology along the dimensions of Gaussian vs. heavy-tailed degree distributions. We hypothesized that heavy-tailed graphs produce more globally synchronized behavior than comparable Gaussian graphs. In accordance with the hypothesis, we find that in a heavy-tailed graph, because of high population synchrony, the difference between neuronal intrinsic properties is minimized, while a Gaussian graph allows read-out of neuronal intrinsic properties. Thus, altering network topology can alter the balance between intrinsically determined vs. synaptically driven network activity.

\section{Data availability}

Underlying data for this study is available from Zenodo. Dataset 1. gscheler/CNeuroSyn: initial version, https://doi. org/10.5281/zenodo.1164096 $6^{14}$.

Data is available under a Creative Commons CC BY-NC 4.0 license.

\section{Software availability}

The source code for the model is available from GitHub: https:// github.com/gscheler/CNeuroSyn/tree/V1.0/src/analysis

Archived source code at time of publication is available from Zenodo https://doi.org/10.5281/zenodo.1164096 ${ }^{14}$.

Software is available under GNU GPL v2.0 license.

\section{Competing interests}

No competing interests were disclosed.

\section{Grant information}

The author(s) declared that no grants were involved in supporting this work.
1. Douglas RJ, Martin KA: Neuronal circuits of the neocortex. Annu Rev Neurosci. 2004: 27(1): 419-451.

PubMed Abstract | Publisher Full Text

2. Douglas RJ, Martin KA: Recurrent neuronal circuits in the neocortex. Curr Biol. 2007; 17(13): R496-500.

PubMed Abstract | Publisher Full Text

3. Carlo CN, Stevens CF: Structural uniformity of neocortex, revisited. Proc Natl Acad Sci U S A. 2013; 110(4): 1488-1493.

PubMed Abstract | Publisher Full Text | Free Full Text

4. Kunze T, Peterson ADH, Haueisen J, et al:: A model of individualized canonical microcircuits supporting cognitive operations. PLOS One. 2017; 12(12): e0188003.

PubMed Abstract | Publisher Full Text | Free Full Text

5. Scheler G: Regulation of neuromodulator receptor efficacy--implications for whole-neuron and synaptic plasticity. Prog Neurobiol. 2004; 72(6): 399-415. PubMed Abstract | Publisher Full Text

6. Scheler G: Learning intrinsic excitability in medium spiny neurons [version 2 ; referees: 2 approved]. F1000Res. 2014; 2: 88 . PubMed Abstract | Publisher Full Text | Free Full Text

7. Izhikevich EM: Which model to use for cortical spiking neurons? IEEE Trans Neural Netw. 2004; 15(5): 1063-1070.

PubMed Abstract | Publisher Full Tex

8. Scheler G: Logarithmic distributions prove that intrinsic learning is Hebbian [version 2; referees: 2 approved]. F1000Res. 2017; 6: 1222. PubMed Abstract | Publisher Full Text | Free Full Text

9. Koulakov AA, Hromádka T, Zador AM: Correlated connectivity and the distribution of firing rates in the neocortex. J Neurosci. 2009; 29(12): 3685-94. PubMed Abstract | Publisher Full Text | Free Full Text

10. Varela JA, Hirsch SJ, Chapman $D$, et al.: $D_{1} / D_{5}$ modulation of synaptic NMDA receptor currents. J Neurosci. 2009; 29(10): 3109-19. PubMed Abstract | Publisher Full Text | Free Full Text

11. Ohshima M, Itami C, Kimura F: The $\alpha_{2 A}$-adrenoceptor suppresses excitatory synaptic transmission to both excitatory and inhibitory neurons in layer 4 barrel cortex. J Physiol. 2017; 595(22): 6923-6937. PubMed Abstract | Publisher Full Text | Free Full Text
12. Kobayashi M, Kojima M, Koyanagi $\mathrm{Y}$, et al:: Presynaptic and postsynaptic modulation of glutamatergic synaptic transmission by activation of alpha ${ }_{1}$ and beta-adrenoceptors in layer $\mathrm{V}$ pyramidal neurons of rat cerebral cortex. Synapse. 2009; 63(4): 269-281.

PubMed Abstract | Publisher Full Text

13. Izhikevich EM, Gally JA, Edelman GM: Spike-timing dynamics of neuronal groups. Cereb Cortex. 2004; 14(8): 933-944. PubMed Abstract | Publisher Full Text

14. Scheler G: gscheler/CNeuroSyn: initial version. Zenodo. 2018 http://www.doi.org/10.5281/zenodo.1164096

15. Barrat A, Barthélemy M, Vespignani A: Dynamical Processes on Complex Networks. Cambridge University Press, 2008. Publisher Full Text

16. Arenas A, Diaz-Guilera A, Kurths J: Synchronization in complex networks. Physics Reports. 2008; 469(3): 93-153. Publisher Full Text

17. Klinshov VV, Teramae JN, Nekorkin VI, et al:: Dense neuron clustering explains connectivity statistics in cortical microcircuits. PLoS One. 2014; 9(4): e94292. PubMed Abstract | Publisher Full Text | Free Full Text

18. Lansner A, Diesmann M: Virtues, Pitfalls, and Methodology of Neuronal Network Modeling and Simulations on Supercomputers. Springer Netherlands, Dordrecht, 2012; 283-315. Publisher Full Text

19. Scheler G, Schumann J: Presynaptic modulation as fast synaptic switching: State-dependent modulation of task performance. In Proceedings of the International Joint Conference on Neural Networks. 2003; 1. Publisher Full Text

20. Packer AM, Yuste R: Dense, unspecific connectivity of neocortical parvalbumin-positive interneurons: a canonical microcircuit for inhibition? J Neurosci. 2011; 31(37): 13260-13271. PubMed Abstract | Publisher Full Text | Free Full Text

21. Graupner M, Reyes AD: Synaptic input correlations leading to membrane potential decorrelation of spontaneous activity in cortex. J Neurosci. 2013; 33(38): 15075-15085.

PubMed Abstract | Publisher Full Text | Free Full Text 
Kruglikov I, Rudy B: Perisomatic GABA release and thalamocortical integration onto neocortical excitatory cells are regulated by neuromodulators. Neuron. 2008; 58(6): 911-924.

PubMed Abstract | Publisher Full Text | Free Full Text

23. Stringer C, Pachitariu M, Steinmetz NA, et al:: Inhibitory control of correlated intrinsic variability in cortical networks. eLife. 2016; 5: pii: e19695. PubMed Abstract | Publisher Full Text | Free Full Text

24. Guedj C, Monfardini E, Reynaud AJ, et al.: Boosting Norepinephrine Transmission Triggers Flexible Reconfiguration of Brain Networks at Rest. Cereb Cortex. 2017; 27(10): 4691-4700. PubMed Abstract | Publisher Full Text

25. Gutnisky DA, Beaman C, Lew SE, et al: Cortical response states for enhanced sensory discrimination. eLife. 2017; 6: pii: e29226. PubMed Abstract | Publisher Full Text | Free Full Text

26. Zylberberg J, Pouget A, Latham PE, et al:: Robust information propagation through noisy neural circuits. PLOS Comput Biol. 2017; 13(4): e1005497. PubMed Abstract | Publisher Full Text | Free Full Text

27. Sompolinsky $\mathrm{H}$, Yoon $\mathrm{H}$, Kang $\mathrm{K}$, et al:: Population coding in neuronal systems with correlated noise. Phys Rev E Stat Nonlin Soft Matter Phys. 2001; 64(5 Pt 1): 051904

PubMed Abstract | Publisher Full Text

28. Goard M, Dan Y: Basal forebrain activation enhances cortical coding of natural scenes. Nat Neurosci. 2009; 12(11): 1444-1449. PubMed Abstract | Publisher Full Text | Free Full Text

29. Edeline JM: Beyond traditional approaches to understanding the functional role of neuromodulators in sensory cortices. Front Behav Neurosci. 2012; 6: 45 PubMed Abstract | Publisher Full Text | Free Full Text

30. Lee SH, Dan Y: Neuromodulation of brain states. Neuron. 2012; 76(1): 209-222. PubMed Abstract | Publisher Full Text | Free Full Text

31. Minces V, Pinto L, Dan Y, et al.: Cholinergic shaping of neural correlations. Proc Natl Acad Sci U S A. 2017; 114(22): 5725-5730. PubMed Abstract | Publisher Full Text | Free Full Text

32. Jeanne JM, Sharpee TO, Gentner TQ: Associative learning enhances population coding by inverting interneuronal correlation patterns. Neuron. 2013; 78(2): 352-363. PubMed Abstract | Publisher Full Text | Free Full Text

33. Fazlali Z, Ranjbar-Slamloo Y, Adibi M, et al.: Correlation between Cortical State and Locus Coeruleus Activity: Implications for Sensory Coding in Rat Barrel Cortex. Front Neural Circuits. 2016; 10: 14.

PubMed Abstract | Publisher Full Text | Free Full Text

34. Harris KD, Thiele A: Cortical state and attention. Nat Rev Neurosci. 2011; 12(9): 509-523.

PubMed Abstract | Publisher Full Text | Free Full Text

35. Schölvinck ML, Saleem AB, Benucci A, et al:: Cortical state determines global variability and correlations in visual cortex. J Neurosci. 2015; 35(1): 170-8. PubMed Abstract | Publisher Full Text | Free Full Text

36. Renart A, de la Rocha J, Bartho P, et al:: The asynchronous state in cortical circuits. Science. 2010; 327(5965): 587-590.

PubMed Abstract | Publisher Full Text | Free Full Text

37. Beaman CB, Eagleman SL, Dragoi V: Sensory coding accuracy and perceptual performance are improved during the desynchronized cortical state. Nat Commun. 2017; 8(1): 1308

PubMed Abstract | Publisher Full Text | Free Full Text

38. Mitchell JF Sundberg KA, Reynolds JH: Spatial attention decorrelates intrinsic activity fluctuations in macaque area V4. Neuron. 2009; 63(6): 879-888. PubMed Abstract | Publisher Full Text | Free Full Text

39. Gutnisky DA, Dragoi V: Adaptive coding of visual information in neural populations. Nature. 2008; 452(7184): 220-4.

PubMed Abstract | Publisher Full Text

40. Kanamaru T, Fujii H, Aihara K: Deformation of attractor landscape via cholinergic presynaptic modulations: a computational study using a phase neuron model. PLoS One. 2013; 8(1): e53854.

PubMed Abstract | Publisher Full Text | Free Full Text

41. Cohen MR, Kohn A: Measuring and interpreting neuronal correlations. Nat Neurosci. 2011; 14(7): 811-819.

PubMed Abstract | Publisher Full Text | Free Full Text

42. Cohen MR, Newsome WT: Context-dependent changes in functional circuitry in visual area MT. Neuron. 2008; 60(1): 162-173. PubMed Abstract | Publisher Full Text | Free Full Text

43. Poulet JF, Petersen CC: Internal brain state regulates membrane potential synchrony in barrel cortex of behaving mice. Nature. 2008; 454(7206): 881-885. PubMed Abstract | Publisher Full Text 


\section{Open Peer Review}

\section{Current Peer Review Status: ? ?}

\section{Version 1}

Reviewer Report 24 October 2018

https://doi.org/10.5256/f1000research.17251.r38207

(C) 2018 Drion G. This is an open access peer review report distributed under the terms of the Creative Commons Attribution License, which permits unrestricted use, distribution, and reproduction in any medium, provided the original work is properly cited.

\section{Guillaume Drion}

Department of Electrical Engineering and Computer Science, University of Liege, Liège, Belgium

This paper compares (i) the response of heterogeneous neurons to two different types of synaptic inputs (uncorrelated or correlated), and (ii) the response of neuronal populations having two different types of synaptic weight distribution (Gaussian or Lognormal). The main results can be summarized as follows:

1. The chosen neuronal heterogeneity induces variability in mean spiking frequency. This variability persists under the application of uncorrelated synaptic inputs, but is masked by the application of correlated synaptic inputs.

2. Stimulating a subpopulation of neurons in a neuronal population induces an asynchronous response when the distribution of synaptic weights is Gaussian, whereas it induces a synchronous response when this distribution is Lognormal.

Then, it is speculated that switches between asynchronous and synchronous states can be controlled by neuromodulators, which would modify the network topology from Gaussian to Lognormal and conversely.

After carefully reading this paper, I think that neither the content nor the form is in a sufficiently advanced stage for the manuscript to be indexed in its current form. A thorough revision is strongly suggested to boost the potential impact of the research. I provide specific comments below, in order of appearance in the text.

Table and figure captions are not descriptive enough. One cannot fully comprehend the different figures, which include numerous notations, by reading the captions. Some notations are not consistent (in Fig. 1-6, ISI is noted I=*** on the left panels, and ISI on the right panels).

Title: The title is totally misleading. It states that "neuromodulation influences and intrinsic readout", but nothing of the sort is demonstrated in the manuscript. Neuromodulation is only speculated to be a plausible source to control the mechanisms highlighted in this manuscript, without any evidence or results to support this speculation. I have nothing against speculations and interpretations, quite the contrary, but they should not appear as grand truth in the title. 
Abstract: The "Methods" part of the abstract is solely composed of results. This part should summarize the methods used in this paper.

\section{Methods:}

"For the experiments in this paper, we only focus on variability induced by changes in the strength of the slow A-type K channels. The total current contribution for this channel is $\mu$ IAs where $\mu$ was selected between 1.0 and 1.5." Please motivate this choice (both channel type and chosen range), because the model is composed of 6 voltage-gated channels that could be used to model variability. Also, I would not use "experiments" but "simulations", although this is a minor comment.

"Time-dependence is modeled by the gating variable u." What does this sentence mean? Both ODEs model time-dependent evolution.

"By varying $d$, we can vary the inactivation dynamics of the model after a spike, by varying a we vary the inactivation dynamics throughout the computation." I do not see the why variables $a$ and $d$ correspond to inactivations. $a$ is the time constant of activation/deactivation of $u$ ( $u$ indeed activates/deactivates, the slope $b$ being always positive), and $d$ is the increase in $u$ after a reset, which represents how much a spike activates $u$.

"For the excitatory neurons, we use randomly connected graphs $(N, K)$ with different dispersion sigma*. This corresponds to normal (Gaussian) to lognormal graphs with different widths and length of the heavy tail.". This dispersion coefficient, and how it "corresponds to normal (Gaussian) to lognormal graphs with different widths and length of the heavy tail ", should be defined properly, because it is an important parameter in the results.

"The rewiring algorithm used to change the properties of a graph $G$ is a greedy algorithm, which iteratively selects the node s with the highest degree". Does "s" refer to a node, or is it a measure of network synchronization?

\section{Results:}

"We show how we can model gain as a stored intrinsic property, defined as a spike rate in response to constant or fluctuating input of fixed strength $(\mathrm{A} / \mathrm{Hz})$ " This sentence is unclear. What kind of gain is it referring to? What is a "stored" intrinsic property? What does "fixed strength" mean for a fluctuating input?

"In Figure 1A, we show the response of MSN model neurons with a scaling of $\mu$ IAs $=1.0,1.3,1.5$ to a noisy signal, derived from uncorrelated Poisson-distributed synaptic input." From what I understood, neurons are not connected to each other in this case. It should be explicitly mentioned, because at this stage it is unclear whether we discuss isolated neurons or neurons within a network.

"The total number of spikes includes bursts, which were excluded from ISI calculation." How were bursts defined? Why were they excluded from ISI calculation? But then why excluding them from one measure but not from the other?

Results related to Fig. 1 and 2: Only one example of uncorrelated/correlated input is shown, and conclusion is drawn from this example. This part requires further investigation. For instance, the increase in firing rate that we see in Fig. 2 could potentially induce overlapping of spiking rate, 
because it moves the neurons to a flatter portion of the f-I curve. It is important to rule out this effect to make sure that the difference is indeed due to the correlated/uncorrelated nature of the inputs, for instance by showing that increasing the firing rate with uncorrelated synaptic inputs does not induce the same effect. I also do not find surprising that inputs with high amplitude, low frequency fluctuations would affect the firing more than an almost constant input, but I guess that it is more subjective. You also talk about "a time-locked spike pattern". How do you define it in the context of this paper? If is it synonymous of synchronization, having clearly separated frequencies does not mean neurons cannot be synchronized: one neuron could have a frequency that is twice the other and be fully synchronized, yet the frequencies would be separated. On the other hand, two neurons could fire at a same frequency and coefficient of variation, and yet not be synchronized.

"To continue with exploring this property of model neurons in a networked context, we switched to a simplified model neuron7 and created a set of variations for this model (see Methods)." Again, I guess that the results of this section are provided on isolated neurons. If it is the case, this sentence is misleading.

Results connected to Fig. 3, 4 and 5: My comment connects to the one relates to Fig. 1 and 2. The spikes of Fig. 3A look much more "time-locked" than the ones of Fig. 2A, although the conclusions that are drawn from these two figures are opposite. Is the "clear separation of ISIs" such a good measure in this context? In Fig. 4 and Fig. 5, most events look like bursts. Are bursts excluded from ISI calculation like in the previous case? If not why? Again, how is bursting defined in this case as compared to the previous case? Finally, in Fig. 5 all neurons seem to burst, yet the distribution of ISIs is a Gaussian, which does not make sense, unless you are only plotting the distribution for the interburst intervals.

"We first use a Gaussian connected graph (RG) with $N=210$ and $K=1800$ and use 7 different neuronal types (1-6, plus the generic neuron $g$ ) with 30 Neurons each (Methods). Figure 7A shows an excerpt of the graph structure. We can see that the graph is connected such that all neurons have a comparable number of connections. This is also apparent in Figure 8, where we can see a (narrow) normal distribution for connectivity for the Gaussian graph RG. Table 2 contains the graph characteristics for both graphs." Only one graph is mentioned in the paragraph, and Table 2 sows 5 different cases. Both graph probably mean "Gaussian" and "Lognormal", but that should be clarified.

"We now stimulate the graph by an initial stimulation to 10 excitatory neurons (for about 1s)." How were these 10 neurons selected? Where they selected randomly, or based on their degree of connectivity? Are they chosen to correspond to nodes with a high connectivity degree in the lognormal case? If so, then what is happening if we either stimulate neurons with lower connectivity degrees in the lognormal case, or add a few hub neurons in the Gaussian case? Is the difference in synchronization really due to the whole distribution of the synaptic weights, or simply due to the existence of hub neurons that would be specifically targeted by synaptic inputs? This question needs to be answered in order to support the conclusion "In our model, the pairwise synchronicity s is dominated by the network topology, more precisely by the width of the degree distribution (dispersion) ranging from Gaussian to lognormal with a heavy tail."

In Figure 11, are the "experimental conditions" similar than in Figures 9 and 10? Which 10 neurons are stimulated?

\section{Discussion}


"Neuromodulation disables or enhances various ion channels, such as SK-channels which guide reset times after a spike, or A-type potassium channels which influence latency to spike." References that support these claims are needed.

Is the work clearly and accurately presented and does it cite the current literature? Partly

Is the study design appropriate and is the work technically sound? Partly

Are sufficient details of methods and analysis provided to allow replication by others? No

If applicable, is the statistical analysis and its interpretation appropriate? Not applicable

Are all the source data underlying the results available to ensure full reproducibility? Yes

Are the conclusions drawn adequately supported by the results? No

Competing Interests: No competing interests were disclosed.

I confirm that I have read this submission and believe that I have an appropriate level of expertise to confirm that it is of an acceptable scientific standard, however I have significant reservations, as outlined above.

Reviewer Report 28 August 2018

\section{https://doi.org/10.5256/f1000research.17251.r37164}

(C) 2018 Zylberberg J. This is an open access peer review report distributed under the terms of the Creative Commons Attribution License, which permits unrestricted use, distribution, and reproduction in any medium, provided the original work is properly cited.

Joel Zylberberg

Department of Physiology and Biophysics, University of Colorado School of Medicine, Aurora, CO, USA

This study asks when the intrinsic biophysical properties of neurons do or do not affect their spiking responses to synaptic inputs. I denote the main claims and results of the paper as I,II,and III below, each of which is discussed individually thereafter.

I. The author begins by noting that, in the presence of strong synchronous inputs, essentially all neurons are driven to quickly spike, regardless of their intrinsic properties. In the presence of less- 
synchronous inputs, differences in intrinsic properties do lead to noticeable changes in spiking outputs. This is (presumably) because differences in integration time, and excitability (effective spiking threshold) determine how much input is needed to get the cell to spike, but sufficiently strong synchronous inputs always suffice to generate spikes. This is a neat observation, somewhat obvious in hindsight, but nevertheless made me glad to read the paper.

II. Next, they investigate how different network structures (quantified by dispersion of the graph's out-degree distribution) affect the synchrony in the inputs to individual neurons: networks with high dispersion (e.g., small numbers of critical "hubs" in the network) lead to higher synchrony. Thus, in those networks, the neurons' intrinsic properties are less influential than in networks with lower dispersion.

III. Finally, the authors speculate that, because neuromodulators can change effective connectivity, they could switch the network between different graph structures, thereby either enabling, or disabling, the influences of neurons' intrinsic properties. In that way, the network can "multiplex" between operational modes, on of which (the asynchronous one) is more affected by neurons' intrinsic plasticity, and thus more affected by memory and experience.

Below, I summarize first the relation to prior studies for each claim (I-III), and then comment on some technical and presentation issues that, to me, hinder somewhat the readability of the paper. My hope is that addressing these concerns will help the author to increase the impact of their work.

\section{Comments on relation to other studies:}

Result I. This is a neat observation. It is somewhat foreshadowed by some older work by Salinas and Sejnowski ${ }^{1,2}$, showing that synchronous inputs are good at driving spikes in basically any neuron model. It would be useful to differentiate the findings of this paper from their older work.

Result II. Closely related results were obtained in a pair of studies by $\mathrm{Yu} \mathrm{Hu}$, James Trousdale, and colleagues ${ }^{3,4}$. There, they computed the correlation structure (closely related to the author's synchrony measure) in networks with different connectivity motifs, using a neat analytical approach involving motif cumulants. It would be worthwhile (again) differentiating the results here from that prior work, so that readers know what's new and significant about this paper vs. the prior state of the field.

Result III. I'm puzzled by the claim that the network topology is strongly affected by neuromodulators (which is the key to the switching property that's discussed through the paper). While I understand that neuromodulators can strengthen or weaken synapses, that mechanism will change the weights within the graph describing network connectivity, but not change the underlying unweighted graph describing which neurons are connected to each other (regardless of the strength of that connection). To change the network topology, the neuromodulators would need to either a) add new synapses (or "unsilence" previously silenced synapses) selectively to some neurons, or b) remove (or completely silence) synapses selectively from some neurons. I think that, in order for the author to make claims about neuromodulators changing network topology, they should include some discussion (ideally based on the experimental literature) of when and how neuromodulators actually change network topology. This literature could of course exist: there's lots that I don't know about neuromodulators. And in 
that case, I think the author's claims would be much strengthened by referencing and discussing it.

Comments on presentation and specific technical issues:

1. Methods, 3rd paragraph. Why only focus on modulators affecting slow A-type K channels? Are those the most important ones? The ones most affected by common neuromodulators? Which neuromodulators are those?

2. Methods, 4th paragraph. It would help me if you would describe explicitly the method used to generate either correlated or uncorrelated synaptic input traces. For example, do these incoming spike trains (generating the synaptic inputs) come from a process like SIP or MIP? And specifically, how were the "correlation factors" quantified and manipulated?

3. Similar to (2) it would help to describe explicitly the synapse model used. For example, are the synapses conductance based (e.g., convolve incoming spike trains with a double exponential to get conductance traces)? I assume not, given that in Figs. 1-6, the same synaptic current trace is shown for all neurons. If the synapses were conductance-based, the differences in membrane potentials of those neurons would lead to differences in synaptic currents.

4. The definition of the synchronization is fine to me but doesn't seem to match the description in the preceding paragraph. Specifically, the formula doesn't seem to "count the number of other neurons which fire in a $10 \mathrm{~ms}$ window. .. " so much as "count the number of spikes emitted by each other neuron in a 10 ms window".

5. I'd describe the rewiring process in the "graph properties" section of the Methods and not the "Definitions" section. That way, when you introduce the dispersion Isigma, you can explain the process used to manipulate it in your simulations.

6. The figures generally could use much more annotation, to help the readers parse what all the different curves mean. For example, in Figs. 1 and 2, I'd color code the curves (distributions) in panel $B$, and have those same colors used for the different spike trains in panel A, so that readers know which distribution goes with which spike train goes with which model. The same idea could be applied to the other figures: help the reader out so that, at a quick glance (which is how people read papers now) they can tell what's what and what the main point is.

Similarly, in Fig. 11, I'd label the color bar, so that people know what variable the colors are describing.

7. The formula for density on p. 6 (bottom left) seems incorrect, as $d=K /(N x N-1)$. That would be $\mathrm{K} /\left(\mathrm{N}^{\wedge} 2-1\right)$, whereas I think you mean $\mathrm{K} /[\mathrm{Nx}(\mathrm{N}-1)]$.

8. P. 6, upper right, description of stimulating the graph by driving 10 excitatory neurons. How was this done? Did you inject current into those model neurons? If so, how much?

9. Some consistency between the network sizes in the simulations would help a lot. Specificlaly, in Fig. 10, you study a 1924 neuron network, whereas Fig. 9 uses 1800 neurons. 
This leave the interpretation muddier than it needs to be: readers might wonder whether some of the differences could be attributed to changes in network size, and not just to changes in connectivity. In general, I'd suggest keeping all but one key variable constant, so that the comparisons can be made as cleanly as possible.

\section{References}

1. Salinas E, Sejnowski T: Impact of Correlated Synaptic Input on Output Firing Rate and Variability in Simple Neuronal Models. The Journal of Neuroscience. 2000; 20 (16): 6193-6209 Publisher Full Text

2. Salinas E, Sejnowski TJ: Correlated neuronal activity and the flow of neural information.Nat Rev Neurosci. 2001; 2 (8): 539-50 PubMed Abstract | Publisher Full Text

3. Trousdale J, Hu Y, Shea-Brown E, Josić K: Impact of network structure and cellular response on spike time correlations.PLoS Comput Biol. 2012; 8 (3): e1002408 PubMed Abstract | Publisher Full Text

4. Hu Y, Trousdale J, Josić K, Shea-Brown E: Local paths to global coherence: cutting networks down to size.Phys Rev E Stat Nonlin Soft Matter Phys. 2014; 89 (3): 032802 PubMed Abstract | Publisher Full Text

Is the work clearly and accurately presented and does it cite the current literature? Partly

Is the study design appropriate and is the work technically sound?

Partly

Are sufficient details of methods and analysis provided to allow replication by others? No

If applicable, is the statistical analysis and its interpretation appropriate? Not applicable

Are all the source data underlying the results available to ensure full reproducibility? Yes

Are the conclusions drawn adequately supported by the results? Partly

Competing Interests: No competing interests were disclosed.

Reviewer Expertise: Computational neuroscience, dynamical systems, sensory systems, memory, learning

I confirm that I have read this submission and believe that I have an appropriate level of expertise to confirm that it is of an acceptable scientific standard, however I have significant reservations, as outlined above. 
The benefits of publishing with F1000Research:

- Your article is published within days, with no editorial bias

- You can publish traditional articles, null/negative results, case reports, data notes and more

- The peer review process is transparent and collaborative

- Your article is indexed in PubMed after passing peer review

- Dedicated customer support at every stage

For pre-submission enquiries, contact research@f1000.com 